\title{
Emergy Evaluation of a Dairy-mushroom Ecosystem in Miyun Reservoir Catchment of Beijing
}

\author{
Yanxia $\mathrm{Hu}$ \\ Institute of Integrated Development of Agriculture, Beijing Academy of Agriculture and Forestry Sciences, Haidian, Beijing, \\ 100097 \\ E-mail:huyx2002@126.com
}

\begin{abstract}
An ecological energetic evaluation is presented in this paper for the dairy-mushroom system in Beijing Miyun reservoir catchment in the year 2010. It developed a system diagram for the dairy and mushroom productions based on Odum's unified measure. Conventional systems indices of the emergy yield ratio (EYR), emergy investment ratio(EIR), environmental load ratio(ELR) and environmental sustainability index(ESI) are introduced for congregated systems ecological assessment. The overall diary-mushroom system is shown with outstanding production competence compared with other agricultural systems in other regions. The mushroom subsystem is highly benefited from the cow subsystem by making use of cow excrements as materials, which can alleviate pollution caused by excessive piles up of cow manure and rain washing down to the groundwater. Compared with the subsystems, diary-mushroom agrocirculation system has higher yield raio, lower emergy investment ratio (EIR), and higher environmental sustainable index (ESI) value. In the long run, processes with a lower percent of purchased emergy are likely to be more sustainable, of which corn plantation, cow breeding and mushroom cultivation are not aparted and formed into a circulation chain. So the dariymushroom circulation system is one of a sustainable agricultural mode adapted for this water protection area and should be promoted.
\end{abstract}

Keywords-emergy evaluation; diary-mushroom system; beijing miyun reservoir catchment.

\section{INTRODUCTION}

In many parts of Beijing suburb, especially in the water protection areas, it has been suffered a great deal profound transformations for the environment between exploitation patterns during the past century [1]. Irrational exploitation patterns are considered to be not only deteriorating the local environments but also reducing the sustainability of agricultural systems. Livestock production system near the reservoir is one of the argumentative exploitation patterns, in light of their manure disposal and environmental impact. Dairy-mushroom system which can use surplus cow manure, decrease water environments impact, is considered to be better exploitation pattern. It is highly in need for an integrated approach to quantify the impact on environmental resources, economic investment and human labor of the dairy-mushroom system in Miyun reservoir with regard to local development and Beijing ecological security.

We often use emergy analysis method to evaluate of the energy efficiency and potentials of the agricultural waste[2,3], and to assess the agricultural systems with different scales[4,5]. Normally, via mainstream monetary approach, extensive dairy-mushroom system analyses has been conducted, aiming to reveal the economics beneficial result. But ecological economic evaluations based on the unified measure of emergy on small scales of family and county associated with the dairy-mushroom system is highly in need.

This study presents an emergy assessment of the overall dairy-mushroom system and its subsystems taking a village in Beijing Miyun Reservoir Catchment (abbreviated as BMRC) as a case study. The objectives of this paper are: (1) to calculate the emergy flows and related indices and ratios of dairy-mushroom system and its subsystems in BMRC. (2) to analyze the ecological sustainability of these systems compared with agricultural systems of other regions, and to reveal the suitability of agricultural exploitation styles as dairy-mushroom system to the local environment considering the ecological pressure exerted by the productions and manures and waste sticks. (3) to explore the implications for the local sustainability and Beijing water security.

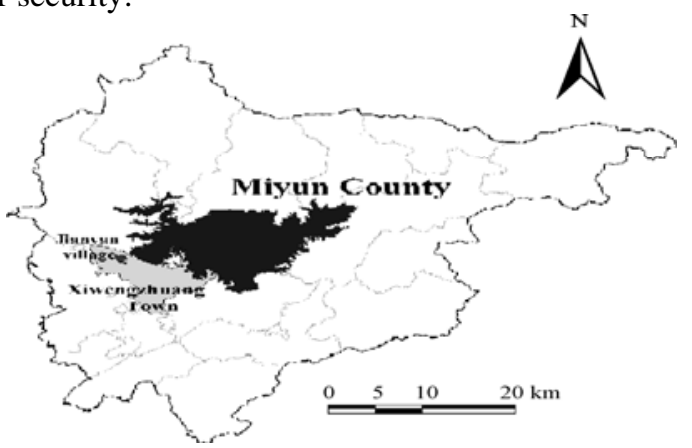

Figure 1. Map of Beijing Miyun Reservoir Catchment Region and location of Jianyan Village in Miyun County 


\section{DAIRY-MUSHROOM SYSTEM IN THE AREA OF BMRC}

Beijing Miyun Reservoir Catchment, as shown in Fig. 1 with a total area of $224 \mathrm{~km}^{2}$, lies in northeast Beijing $\left(40^{\circ}\right.$ $30^{\prime} \mathrm{E}-117^{\circ} 10^{\prime} \mathrm{N}$ ), total water capability is 4375 million $\mathrm{m}^{3}$ [6]. It was built in 1960, since then, Miyun reservoir is the major hydraulic engineering in north China with function of flood control, irrigation, water supply, electricity generating, fish culture and tour, et al. All of which decrease gradually to one function of supply drinking water to Beijing city solely due to the shortage of water resource since 1980, and thereupon environmental protection of this region is very important for the sustainability of Beijing city [7].

The whole catchment are divided into three protecting areas, the first and the second areas are located in Miyun county, of which there are about 50 villages. It's often have a contradiction between economical development and environmental protection. Jianyan village, as shown in Fig. 1 with a total area of $5.2 \mathrm{~km}^{2}$ and 1100 farmers, lies in Xiwengzhuang Town, Miyun county[8]. There are a dairy farm raised about 350 cows and an edible mushroom plant which produced in 25 greenhouses and 20 overhead-sheds. Meanwhile there are $100 \mathrm{hm}^{2}$ corn fields and $167 \mathrm{hm}^{2}$ orchards. Diary farm supplies milk for market, provides organic manure for the mushroom, fed by crop residues in and out of the village. Mushroom plant used cow manure and waste branches to produce mushrooms and other waste sticks, some of them are the part of corn fertilizer. So diarymushroom system formed a whole circulation system, aimed to decrease excretion manure to the environment and produce more economic benefits. This circulation model maybe the one we should allocate for protecting water environment, and highly in need to quantify its ecological and economic influences.

\section{SYSTEM DIAGRAM AND EMERGY ANALYSIS}

We draw an aggregated diagram for the diarymushroom system to illustrate the material and energy flows and the organization of major components that utilize those resources [5], the dairy-mushroom system are driven by natural resources and economic investments. Associated with this diagram, inputs to the dairy-mushroom systems might be categorized into four types: free renewable local resources (RR), such as sunlight, rain and wind; free nonrenewable local resources (NR); non-renewable purchased inputs (NP), such as purchased chemical fertilizers; and renewable purchased inputs (RP), such as forage purchased from outside the boundary of the concerned system or subsystem.

Emergy yield ratio $\mathrm{EYR}=\mathrm{Y} /(\mathrm{NP}+\mathrm{RP})$

Emergy investment ratio $\mathrm{EIR}=(\mathrm{NP}+\mathrm{RP}) /(\mathrm{RR}+\mathrm{NR})(2)$

Environmental load ratio $E L R=(N P+N R) /(R R+R P)(3)$

Environmental sustainability ESI=EYR/ELR

Jianyan in BMRC In this study, most of data, especially the economic inputs of goods and service and the yields, are taken primarily from field survey and experimental project or get a estimation by appropriate method. Some types of data are from official statistics of Miyun County and some experimental reports. As one year is taken as the time scale for the present analysis of the diary-mushroom system in the year of 2000, all buildings and tools used in the production systems are converted to annual flows based on the local investigation.

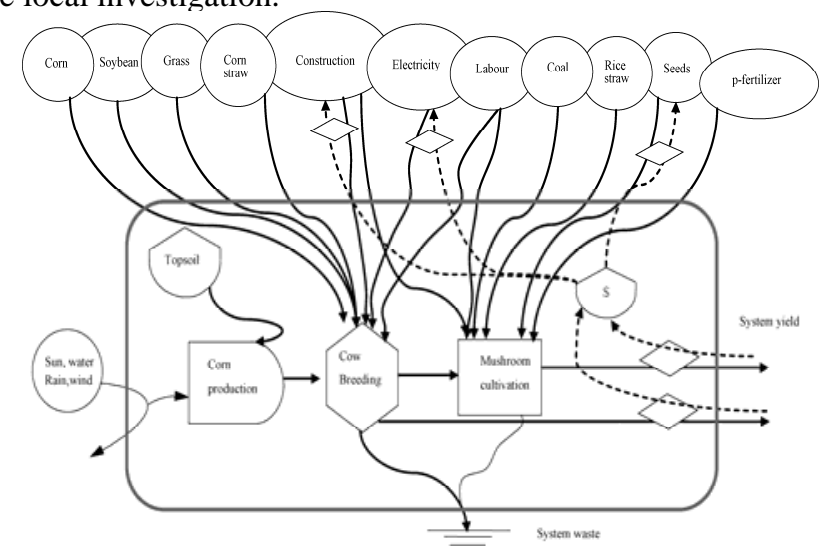

Figure 2. Diagram of aggregated energy and material flows for the cowmushroom system on Jianyan farm

\section{RESUlt AND CONCLUSIONS}

Ecological energetic evaluations based upon Odum's concept of emergy as embodied solar energy are conducted on the overall system and subsystems of Beijing Miyun Reservoir Catchment in northern China to identify their production efficiency and environmental sustainability. We use conventional systems indices of EYR, EIR, ELR and ESI to assess the congregated systems.

Extensive emergy account has been made for the concerned systems and subsystems, and concrete conclusions are drawn as follows:

(1) The whole dairy-mushroom system is estimated to be supported by total emergy of $6.94 \times 10^{18}$ sej yr-1 in 2010 . The operation of the mushroom and cow

production heavily relies on outer investments with $78.67 \%$ of the total input $\left(5.46 \times 10^{18}\right.$ sej $\left.y r-1\right)$ of which the largest emergy inflow is materials such as corn, soybean and straw accounting for $26.94 \%, 22 \%$ and $18.04 \%$ of total input respectively. High outside economy input ratio reflect higher development characteristics of outer environment (Table 1).

(2) The overall dairy-mushroom system is shown with outstanding production competence compared with other agricultural systems such as catfish system in USA, aquaculture farm system in Beijing Miyun, as indicated by ESI value of 10.94 for the dairy-mushroom system in Miyun reservoir catchment against 0.42, 0.22, 0.22 for aquaculture farm system and planting system, respectively (Table 4).

(3) The mushroom subsystem is highly benefited from the cow subsystem by making use of cow excrements as materials(Table 2). The use intensity of manure is 1.46 
TABLE I. EMERGY EVALUATION TABLE FOR OVERALL DIARY -MUSHROOM CIRCULATION ECOSYSTEM IN BMRC

\begin{tabular}{|c|c|c|c|c|c|c|c|c|c|c|c|}
\hline Resource & Quantity & Unit & $\begin{array}{l}\text { Energy } \\
\text { conversion } \\
\text { factor }\end{array}$ & Unit & $\begin{array}{l}\text { Energy or } \\
\text { material } \\
\text { flow }\end{array}$ & Unit & Transformity & Unit & Ref. & $\begin{array}{l}\text { Emergy } \\
\text { (Sej/year) }\end{array}$ & $\%$ \\
\hline \multicolumn{12}{|c|}{ Renewable resources from free environment (RR) } \\
\hline Sunlight & $4.67 \mathrm{E}+04$ & $\mathrm{~m} 2$ & $3.30 \mathrm{E}+09$ & $\mathrm{~J} / \mathrm{m}^{2}$ & $1.54 \mathrm{E}+14$ & $\mathrm{~J}$ & 1 & Sej/J & Odum, 2000 & $1.54 \mathrm{E}+14$ & $<0.1$ \\
\hline Rain,chemical & $3.74 \mathrm{E}+03$ & $\mathrm{~m} 3$ & $4.94 \mathrm{E}+06$ & $\mathrm{~J} / \mathrm{m}^{3}$ & $1.85 \mathrm{E}+10$ & $\mathrm{~J}$ & $4.28 \mathrm{E}+05$ & Sej/J & Yan, 2001 & $7.90 \mathrm{E}+15$ & 0.11 \\
\hline Rain,geopotencial & $7.00 \mathrm{E}+05$ & m3 & $4.94 \mathrm{E}+06$ & $\mathrm{~J} / \mathrm{m}^{3}$ & $3.46 \mathrm{E}+12$ & $\mathrm{~J}$ & $4.28 \mathrm{E}+05$ & $\mathrm{Sej} / \mathrm{J}$ & Yan, 2001 & $1.48 \mathrm{E}+18$ & 21.33 \\
\hline Wind & $4.67 \mathrm{E}+04$ & $\mathrm{~m} 2$ & $7.50 \mathrm{E}+08$ & $\mathrm{~J} / \mathrm{m}^{2}$ & $3.50 \mathrm{E}+13$ & $\mathrm{~J}$ & 1496 & Sej/J & Yan, 2001 & $5.24 \mathrm{E}+16$ & 0.75 \\
\hline & & & & & & & & & & $1.48 \mathrm{E}+18$ & 21.33 \\
\hline Loss of topsoil & $4.67 \mathrm{E}+04$ & $\mathrm{~m} 2$ & $8.37 \mathrm{E}+04$ & $\mathrm{~J} / \mathrm{m}^{2}$ & $3.91 E+09$ & $\mathrm{~J}$ & $6.25 \mathrm{E}+04$ & Sej/J & Sherry, 2002 & $2.44 \mathrm{E}+14$ & $<0.1$ \\
\hline \multicolumn{12}{|c|}{ Nonrenewable purchases (NP) } \\
\hline Construction & $3.50 \mathrm{E}+05$ & $¥$ & 7.00 & $¥ / \$$ & 50000.00 & $\$$ & $1.16 \mathrm{E}+13$ & Sej/\$ & Hu, 2008 & $5.80 \mathrm{E}+17$ & 8.35 \\
\hline Coal & $2.00 \mathrm{E}+04$ & $\mathrm{~kg}$ & $2.93 \mathrm{E}+07$ & $\mathrm{~J} / \mathrm{kg}$ & $5.86 \mathrm{E}+11$ & $\mathrm{~J}$ & 39800 & Sej/J & Mark, 2002 & $2.33 \mathrm{E}+16$ & 0.34 \\
\hline P-fertilizer & $3.60 \mathrm{E}+02$ & $\mathrm{~kg}$ & & & & & $6.55 \mathrm{E}+12$ & $\mathrm{Sej} / \mathrm{kg}$ & Mark, 2002 & $2.36 \mathrm{E}+15$ & 0.03 \\
\hline Water & $2.36 \mathrm{E}+08$ & $\mathrm{~kg}$ & $4.94 \mathrm{E}+03$ & $\mathrm{~J} / \mathrm{kg}$ & $1.16 \mathrm{E}+12$ & $\mathrm{~J}$ & $4.28 \mathrm{E}+05$ & Sej/J & Yan, 2001 & $4.98 \mathrm{E}+17$ & 7.18 \\
\hline Electricity & $3.00 \mathrm{E}+04$ & $Y$ & 7.00 & $Y / \$$ & 4285.71 & $\$$ & $1.16 \mathrm{E}+13$ & Sej/\$ & Hu, 2008 & $4.97 \mathrm{E}+16$ & 0.72 \\
\hline Total NP & & & & & & & & & & $1.15 \mathrm{E}+18$ & 16.62 \\
\hline \multicolumn{12}{|c|}{ Renewable subsidiary resources from economy (RP) } \\
\hline Seeds & $9.00 \mathrm{E}+04$ & $¥$ & 7.00 & $¥ / \$$ & $1.29 \mathrm{E}+04$ & $\$$ & $1.16 \mathrm{E}+13$ & Sej/\$ & Sherry, 2002 & $1.49 \mathrm{E}+17$ & 2.15 \\
\hline Labour & $9.20 \mathrm{E}+03$ & person & $1.05 \mathrm{E}+07$ & $\mathrm{~J} /$ person & $9.63 \mathrm{E}+10$ & $\mathrm{~J}$ & $1.24 \mathrm{E}+06$ & Sej/J & Sherry, 2002 & $1.19 \mathrm{E}+17$ & 2.10 \\
\hline Corn & $5.11 \mathrm{E}+05$ & $\mathrm{~kg}$ & $1.65 \mathrm{E}+07$ & $\mathrm{~J} / \mathrm{kg}$ & $8.45 \mathrm{E}+12$ & $\mathrm{~J}$ & $8.52 E+04$ & Sej/J & Sherry, 2002 & $7.20 \mathrm{E}+17$ & 10.37 \\
\hline Soybean & $2.04 \mathrm{E}+05$ & $\mathrm{~kg}$ & $1.85 \mathrm{E}+07$ & $\mathrm{~J} / \mathrm{kg}$ & $3.78 \mathrm{E}+12$ & $\mathrm{~J}$ & $4.04 \mathrm{E}+05$ & Sej/J & Sherry, 2002 & $1.53 \mathrm{E}+18$ & 22.00 \\
\hline Grass & $3.83 E+05$ & $\mathrm{~kg}$ & $1.47 \mathrm{E}+07$ & $\mathrm{~J} / \mathrm{kg}$ & $5.61 \mathrm{E}+12$ & $\mathrm{~J}$ & $4.98 \mathrm{E}+04$ & Sej/J & Sherry, 2002 & $2.80 \mathrm{E}+17$ & 4.03 \\
\hline Straw & $3.19 \mathrm{E}+06$ & $\mathrm{~kg}$ & $1.45 \mathrm{E}+07$ & $\mathrm{~J} / \mathrm{kg}$ & $4.64 \mathrm{E}+13$ & $\mathrm{~J}$ & $2.70 \mathrm{E}+04$ & Sej/J & Sherry, 2002 & $1.25 \mathrm{E}+18$ & 18.04 \\
\hline Total RP & & & & & & & & & & $4.31 \mathrm{E}+18$ & 62.05 \\
\hline Total input & & & & & & & & & & $6.94 \mathrm{E}+18$ & \\
\hline \multicolumn{12}{|l|}{ Yield of System } \\
\hline Milk & $1.70 \mathrm{E}+06$ & $\mathrm{~kg}$ & & & & & $3.33 E+12$ & Sej/kg & & $5.68 \mathrm{E}+18$ & \\
\hline Mushroom & $1.00 \mathrm{E}+05$ & $\mathrm{~kg}$ & & & & & $2.67 \mathrm{E}+13$ & Sej/kg & & $2.67 \mathrm{E}+18$ & \\
\hline Calf & $4.04 \mathrm{E}+10$ & $\mathrm{~J}$ & & & & & $1.86 \mathrm{E}+06$ & Sej/J & & $7.54 \mathrm{E}+16$ & \\
\hline Waste material & $1.60 \mathrm{E}+05$ & $\mathrm{~kg}$ & & & & & $1.67 \mathrm{E}+13$ & Sej/kg & & $1.67 \mathrm{E}+13$ & \\
\hline Manure & $2.15 E+05$ & $\mathrm{~kg}$ & & & & & $1.62 \mathrm{E}+13$ & Sej/kg & & $3.49 \mathrm{E}+18$ & \\
\hline Total Yield & & & & & & & & & & $1.19 \mathrm{E}+19$ & \\
\hline
\end{tabular}

$\times 10^{18}$ sej yr-1, use of cow manure is considered a sustainable strategy for catchment agriculture, which can alleviate pollution caused by excessive piles up of cow manure and rain washing down to the groundwater, which has close relationship to Miyun reservoir water environment. In recent 10 years, because of lack of rain and reduction of reservoir water, about $6667 \mathrm{hm}^{2}$ land emerges from water which local farmers call this as "staked land", according to the government policy, which is not allowable for farmers to cultivate, if cultivate, chemical fertilizer is forbidden. But farmers often cultivate the emerge land to increase their incomes, and take it very trouble to use organic manure compared with buying chemical fertilizers at the cost they could accept, in a word, it's common to use chemical fertilizers to cultivate the emerged land from Miyun reservoir, but not using organic fertilizer. So making full use of cow manure and form agro-circulation system are significance to this water protecting area.
(4) The value of EYR for the dairy subsystem is higher than dairy and mushroom subsystem, the environmental sustainable index (ESI) for the agro-circulation is greater than the both subsystems. Compared with the subsystems, agro-circulation system has higher yield raio, lower emergy investment ratio (EIR)(Table 3), and higher environmental sustainable index (ESI) value(Table 5). In the long run, processes with a lower percent of purchased emergy are likely to be more sustainable than those with a high percent of purchased emergy. So the agro-circulation system is suitable to local situation. In other regions, corn plantation, cow breeding and mushroom cultivation are aparted and not formed into a circulation chain. In Miyun water reservoir catchment, this agro-circulation system should be promoted. So the dariy-mushroom circulation system is one of a sustainable agricultural mode adapted for this water protection area. 
TABLE II. EMERGY INDICES FOR DIARY SUB-SYSTEM COMPARED WITH THOSE OF OTHER AGRICULTURE SYSTEM

\begin{tabular}{|c|c|c|c|c|c|c|c|c|c|c|c|}
\hline Resource & Quantity & Unit & $\begin{array}{l}\text { Energy conversion } \\
\text { factor }\end{array}$ & Unit & $\begin{array}{l}\text { Energy or } \\
\text { material flow }\end{array}$ & Unit & Transformity & Unit & Ref. & $\begin{array}{l}\text { Emergy } \\
\text { (Sej/year) }\end{array}$ & $\%$ \\
\hline \multicolumn{12}{|c|}{ Renewable resources from free environment (RR) } \\
\hline Sunlight & $3.34 \mathrm{E}+04$ & $\mathrm{~m}^{2}$ & $3.30 \mathrm{E}+09$ & $\mathrm{~J} / \mathrm{m}^{2}$ & $1.10 \mathrm{E}+14$ & $\mathrm{~J}$ & 1 & Sej/J & Odum, 2000 & $1.10 \mathrm{E}+14$ & $<0.1$ \\
\hline Rain,chemical & $2.67 \mathrm{E}+03$ & $\mathrm{~m}^{3}$ & $4.94 \mathrm{E}+06$ & $\mathrm{~J} / \mathrm{m}^{3}$ & $1.32 \mathrm{E}+10$ & $\mathrm{~J}$ & $4.28 \mathrm{E}+05$ & $\mathrm{Sej} / \mathrm{J}$ & Yan, 2001 & $5.64 \mathrm{E}+15$ & 0.10 \\
\hline Rain,geopotencial & $5.00 \mathrm{E}+05$ & $\mathrm{~m}^{3}$ & $4.94 \mathrm{E}+06$ & $\mathrm{~J} / \mathrm{m}^{3}$ & $2.47 \mathrm{E}+12$ & $\mathrm{~J}$ & $4.28 \mathrm{E}+05$ & Sej/J & Yan, 2001 & $1.06 \mathrm{E}+18$ & 18.63 \\
\hline $\begin{array}{l}\text { Wind } \\
\text { Total RR }\end{array}$ & $3.34 \mathrm{E}+04$ & $\mathrm{~m}^{2}$ & $7.50 \mathrm{E}+08$ & $\mathrm{~J} / \mathrm{m}^{2}$ & $2.50 \mathrm{E}+13$ & $\mathrm{~J}$ & 1496 & Sej/J & Yan, 2001 & $\begin{array}{l}3.74 \mathrm{E}+16 \\
1.06 \mathrm{E}+18 \\
\end{array}$ & $\begin{array}{l}0.66 \\
18.63 \\
\end{array}$ \\
\hline \multicolumn{12}{|c|}{ Non-renewable resources from free environment (NR) } \\
\hline $\begin{array}{l}\text { Loss of topsoil } \\
\text { Total NR }\end{array}$ & $3.34 \mathrm{E}+04$ & $\mathrm{~m}^{2}$ & $8.37 \mathrm{E}+04$ & $\mathrm{~J} / \mathrm{m}^{2}$ & $2.79 \mathrm{E}+09$ & $\mathrm{~J}$ & $6.25 \mathrm{E}+04$ & Sej/J & Sherry, 2002 & $\begin{array}{l}1.75 \mathrm{E}+14 \\
1.75 \mathrm{E}+14\end{array}$ & $\begin{array}{l}<0.1 \\
<0.1\end{array}$ \\
\hline \multicolumn{12}{|c|}{ Nonrenewable purchases (NP) } \\
\hline Construction & $2.70 \mathrm{E}+05$ & $Y$ & 7.00 & $Y / \$$ & 38571.43 & $\$$ & $1.16 \mathrm{E}+13$ & Sej/\$ & $\mathrm{Hu}, 2008$ & $4.47 \mathrm{E}+17$ & 7.88 \\
\hline Electricity & $3.00 \mathrm{E}+04$ & $¥$ & 7.00 & $¥ / \$$ & 4285.71 & $\$$ & $1.16 \mathrm{E}+13$ & $\mathrm{Sej} / \$$ & Hu, 2008 & $4.97 \mathrm{E}+16$ & 0.88 \\
\hline $\begin{array}{l}\text { Water } \\
\text { Total NR }\end{array}$ & $1.28 \mathrm{E}+08$ & $\mathrm{~kg}$ & $4.94 \mathrm{E}+03$ & $\mathrm{~J} / \mathrm{kg}$ & $6.31 \mathrm{E}+11$ & $\mathrm{~J}$ & $4.28 \mathrm{E}+05$ & Sej/J & Yan, 2001 & $\begin{array}{l}2.70 \mathrm{E}+17 \\
7.67 \mathrm{E}+17 \\
\end{array}$ & $\begin{array}{l}4.76 \\
13.51 \\
\end{array}$ \\
\hline \multicolumn{12}{|c|}{ Renewable subsidiary resources from economy (RP) } \\
\hline Corn & $5.11 \mathrm{E}+05$ & $\mathrm{~kg}$ & $1.65 \mathrm{E}+07$ & $\mathrm{~J} / \mathrm{kg}$ & $8.45 \mathrm{E}+12$ & $\mathrm{~J}$ & $8.52 \mathrm{E}+04$ & Sej/J & Sherry, 2002 & $7.20 \mathrm{E}+17$ & 12.68 \\
\hline Soybean & $2.04 \mathrm{E}+05$ & $\mathrm{~kg}$ & $1.85 \mathrm{E}+07$ & $\mathrm{~J} / \mathrm{kg}$ & $3.78 \mathrm{E}+12$ & $\mathrm{~J}$ & $4.04 \mathrm{E}+05$ & $\mathrm{Sej} / \mathrm{J}$ & Sherry, 2002 & $1.53 \mathrm{E}+18$ & 26.91 \\
\hline Grass & $3.83 \mathrm{E}+05$ & $\mathrm{~kg}$ & $1.47 \mathrm{E}+07$ & $\mathrm{~J} / \mathrm{kg}$ & $5.61 \mathrm{E}+12$ & $\mathrm{~J}$ & $4.98 \mathrm{E}+04$ & Sej/J & Sherry, 2002 & $2.80 \mathrm{E}+17$ & 4.93 \\
\hline Straw & $3.19 \mathrm{E}+06$ & $\mathrm{~kg}$ & $1.45 \mathrm{E}+07$ & $\mathrm{~J} / \mathrm{kg}$ & $4.64 \mathrm{E}+13$ & $\mathrm{~J}$ & $2.70 \mathrm{E}+04$ & $\mathrm{Sej} / \mathrm{J}$ & Sherry, 2002 & $1.25 \mathrm{E}+18$ & 22.06 \\
\hline Labour & $5.60 \mathrm{E}+03$ & person & $1.05 \mathrm{E}+07$ & $\mathrm{~J} /$ person & $5.86 \mathrm{E}+10$ & $\mathrm{~J}$ & $1.24 \mathrm{E}+06$ & $\mathrm{Sej} / \mathrm{J}$ & Sherry, 2002 & $7.27 \mathrm{E}+16$ & 1.28 \\
\hline Total RP & & & & & & & & & & $3.85 \mathrm{E}+18$ & 67.85 \\
\hline Total input & & & & & & & & & & $5.68 \mathrm{E}+18$ & \\
\hline \multicolumn{12}{|l|}{ Yield of System } \\
\hline Milk & $1.70 \mathrm{E}+06$ & $\mathrm{~kg}$ & & & & & $3.33 E+12$ & Sej/kg & & $5.68 \mathrm{E}+18$ & \\
\hline Calf & $4.04 \mathrm{E}+10$ & $\mathrm{~J}$ & & & & & $1.86 \mathrm{E}+06$ & $\mathrm{Sej} / \mathrm{J}$ & & $7.54 \mathrm{E}+16$ & \\
\hline Manure & $3.45 \mathrm{E}+05$ & $\mathrm{~kg}$ & & & & & $1.62 \mathrm{E}+13$ & $\mathrm{Sej} / \mathrm{kg}$ & & $5.60 \mathrm{E}+18$ & \\
\hline
\end{tabular}




\begin{tabular}{|c|c|c|c|c|c|c|c|c|c|c|c|}
\hline Resource & Quantity & Unit & $\begin{array}{l}\text { Energy } \\
\text { conversion } \\
\text { factor }\end{array}$ & Unit & $\begin{array}{l}\text { Energy or } \\
\text { material } \\
\text { flow }\end{array}$ & Unit & Transformity & Unit & Ref. & $\begin{array}{l}\text { Emergy } \\
\text { (Sej/year) }\end{array}$ & $\%$ \\
\hline \multicolumn{12}{|c|}{ Renewable resources from free environment (RR) } \\
\hline Sunlight & $1.33 \mathrm{E}+04$ & $\mathrm{~m}^{2}$ & $3.30 \mathrm{E}+09$ & $\mathrm{~J} / \mathrm{m}^{2}$ & $4.40 \mathrm{E}+13$ & $\mathrm{~J}$ & 1 & Sej/J & Odum, 2000 & $4.40 \mathrm{E}+13$ & $<0.1$ \\
\hline Rain,chemical & $1.07 \mathrm{E}+03$ & $\mathrm{~m}^{3}$ & $4.94 \mathrm{E}+06$ & $\mathrm{~J} / \mathrm{m}^{3}$ & $5.27 \mathrm{E}+09$ & $\mathrm{~J}$ & $4.28 \mathrm{E}+05$ & Sej/J & Yan, 2001 & $2.26 \mathrm{E}+15$ & 0.08 \\
\hline $\begin{array}{l}\text { Rain,geopotencial energy (J) } \\
\text { Wind }\end{array}$ & $\begin{array}{l}2.00 \mathrm{E}+05 \\
1.33 \mathrm{E}+04\end{array}$ & $\begin{array}{l}\mathrm{m}^{3} \\
\mathrm{~m}^{2}\end{array}$ & $\begin{array}{l}4.94 \mathrm{E}+06 \\
7.50 \mathrm{E}+08\end{array}$ & $\mathrm{~J} / \mathrm{m}^{3}$ & $\begin{array}{l}9.88 \mathrm{E}+11 \\
1.00 \mathrm{E}+13\end{array}$ & $\begin{array}{l}\mathrm{J} \\
\mathrm{J}\end{array}$ & $\begin{array}{l}4.28 \mathrm{E}+05 \\
1.50 \mathrm{E}+03\end{array}$ & $\begin{array}{l}\text { Sej/J } \\
\text { Sej/J }\end{array}$ & $\begin{array}{l}\text { Yan, } 2001 \\
\text { Yan, } 2001\end{array}$ & $\begin{array}{l}4.23 \mathrm{E}+17 \\
1.50 \mathrm{E}+16\end{array}$ & $\begin{array}{l}15.84 \\
0.56\end{array}$ \\
\hline Total RR & & & & & & & & & & $4.23 \mathrm{E}+17$ & 15.84 \\
\hline \multicolumn{12}{|c|}{ Non-renewable resources from free environment (NR) } \\
\hline Loss of topsoil & $1.33 \mathrm{E}+04$ & $\mathrm{~m}^{2}$ & $8.37 \mathrm{E}+04$ & $\mathrm{~J} / \mathrm{m}^{2}$ & $1.12 \mathrm{E}+09$ & $\mathrm{~J}$ & $6.25 \mathrm{E}+04$ & Sej/J & Sherry, 2002 & $6.98 \mathrm{E}+13$ & $<0.1$ \\
\hline Total NR & & & & & & & & & & $6.98 \mathrm{E}+13$ & $<0.1$ \\
\hline \multicolumn{12}{|l|}{ Nonrenewable purchases (NP) } \\
\hline Construction & $8.00 \mathrm{E}+04$ & $¥$ & 7.00 & $¥ / \$$ & 11428.57 & $\$$ & $1.16 \mathrm{E}+13$ & Sej/\$ & Hu, 2008 & $1.33 \mathrm{E}+17$ & 4.96 \\
\hline Coal & $2.00 \mathrm{E}+04$ & $\mathrm{~kg}$ & $2.93 \mathrm{E}+07$ & $\mathrm{~J} / \mathrm{kg}$ & $5.86 \mathrm{E}+11$ & $\mathrm{~J}$ & 39800 & Sej/J & Mark, 2001 & $2.33 \mathrm{E}+16$ & 0.87 \\
\hline P-fertilizer & $3.60 \mathrm{E}+02$ & $\mathrm{~kg}$ & & & & & $6.55 \mathrm{E}+12$ & Sej/kg & Mark, 2001 & $2.36 \mathrm{E}+15$ & 0.09 \\
\hline Water & $1.08 \mathrm{E}+08$ & $\mathrm{~kg}$ & $4.94 \mathrm{E}+03$ & $\mathrm{~J} / \mathrm{kg}$ & $5.34 \mathrm{E}+11$ & $\mathrm{~J}$ & $4.28 \mathrm{E}+05$ & Sej/J & Yan, 2001 & $2.28 \mathrm{E}+17$ & 8.55 \\
\hline Total NP & & & & & & & & & & $3.87 \mathrm{E}+17$ & 14.47 \\
\hline \multicolumn{12}{|c|}{ Renewable subsidiary resources from economy (RP) } \\
\hline Rice straw & $3.00 \mathrm{E}+05$ & $\mathrm{~kg}$ & $1.41 \mathrm{E}+07$ & $\mathrm{~J} / \mathrm{kg}$ & $4.22 \mathrm{E}+12$ & $\mathrm{~J}$ & $4.98 \mathrm{E}+04$ & Sej/J & Sherry, 2002 & $2.10 \mathrm{E}+17$ & 7.86 \\
\hline Manure & $1.30 \mathrm{E}+05$ & $\mathrm{~kg}$ & & & & & $1.12 \mathrm{E}+13$ & Sej/kg & Sherry, 2002 & $1.46 \mathrm{E}+18$ & 54.50 \\
\hline Seeds & $9.00 \mathrm{E}+04$ & $¥$ & 7.00 & $¥ / \$$ & $1.29 \mathrm{E}+04$ & $\$$ & $1.16 \mathrm{E}+13$ & Sej/\$ & $\mathrm{Hu}, 2008$ & $1.49 \mathrm{E}+17$ & 5.58 \\
\hline Labour & $3.60 \mathrm{E}+03$ & person & $1.05 \mathrm{E}+07$ & $\mathrm{~J} /$ person & $3.77 \mathrm{E}+10$ & $\mathrm{~J}$ & $1.24 \mathrm{E}+06$ & $\mathrm{Sej} / \mathrm{J}$ & Sherry, 2002 & $4.67 \mathrm{E}+16$ & 1.75 \\
\hline Total RP & & & & & & & & & & $1.86 \mathrm{E}+18$ & 69.69 \\
\hline Total input & & & & & & & & & & $2.67 \mathrm{E}+18$ & \\
\hline \multicolumn{12}{|l|}{ Yield of System } \\
\hline Mushroom & $1.00 \mathrm{E}+05$ & $\mathrm{~kg}$ & & & & & $2.67 \mathrm{E}+13$ & Sej/kg & & $2.67 \mathrm{E}+18$ & \\
\hline Waste material & $1.60 \mathrm{E}+05$ & $\mathrm{~kg}$ & & & & & $1.67 \mathrm{E}+13$ & Sej/kg & & $2.67 \mathrm{E}+18$ & \\
\hline
\end{tabular}




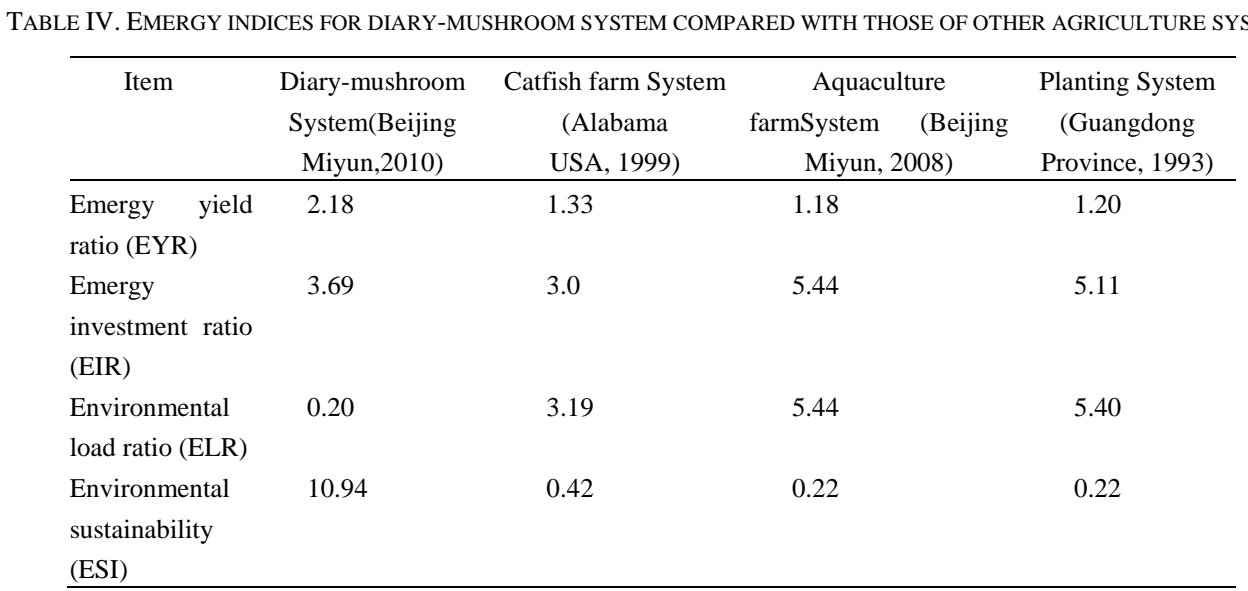

Table V. Results of Multiple Comparisons In Soil NO3-N Content With LSD Method (With MeAn Values of pH ) in EAch LAND Use Type

\begin{tabular}{llll}
\hline Emergy indicators & $\begin{array}{l}\text { Diary breeding } \\
\text { subsystem }\end{array}$ & Mushroom cultivation subsystem & $\begin{array}{l}\text { Agro-circulation } \\
\text { system }\end{array}$ \\
\hline $\begin{array}{l}\text { Emergy yield ratio } \\
\text { EYR=Y/(NP+RP) }\end{array}$ & 1.23 & 1.19 & 2.18 \\
\hline $\begin{array}{l}\text { Emergy investment } \\
\text { ratio }\end{array}$ & 4.37 & 5.31 & 3.69 \\
$\begin{array}{l}\text { EIR= (NP+RP ) } \\
\text { (RR+NR) }\end{array}$ & & 0.17 & 0.20 \\
\hline $\begin{array}{l}\text { Environmental } \\
\text { loading ratio } \\
\text { ELR= (NP+NR) } \\
(\mathrm{RP}+\mathrm{RR})\end{array}$ & 0.16 & & 10.94 \\
\hline $\begin{array}{l}\text { Renewability(\%) } \\
\text { ESI=EYR/ELR }\end{array}$ & 7.86 & 7.02 & \\
\hline
\end{tabular}

\section{V.ACKNOWLEDGEMENT}

Project supported by the Beijing Natural Science foundation(8132028)

\section{REFERENCES}

[1] Wang Q.S., Mei X.R., Zhang Y.Q.2009, Review of water quality of Miyun reservoir. Journal of agricultural science and technology, 1, 45-50.

[2] Seksan Papong, Tassaneewan Chom-in, Soottiwan Noksa-nga et al., 2010. Life cycle energy efficiency and potentials of biodiesel production from palm oil in Thailand, 38,226-233.

[3] John A. Mathews, Hao Tan, Michael J.B et al., 2011. A conceptual lignocellulosic 'feed +fuel' biorefinery and its application to the linked biofuel and cattle raising industries in Brazil, 39,4932-4938.

[4] Chen, G.Q., Jiang, M.M., Chen, B., Yang, Z.F., Lin, C.,2006. Emergy analysis of Chinese agriculture. Agriculture, Ecosystems and Environment 115,161173.

[5] Martin, J.F., Diemont, A.W., Powell, E., 2006. Emergy evaluation of the performance and sustainability of three agricultural systems with different scales and management. Agriculture, Ecosystems and Enviroment,115,128-140.

[6] Hu,Y.X., Zhou L.D., Yan M.C., 2007. The valuation and compensation policies suggestions of main capitals and services of Miyun reservoir[J]. Journal of Natural Resources 4,497-506(in Chinese).
[7] Yin J.Z., Yu T., Wang X.Y., 2009. Discharge features of rural domestic wastewater from different types of villages in water source protection area, Miyun reservoir, Beijing[J]. Journal of Agro-Environment Science, 6,1200 $-1207$.

[8] Hu,Y.X., Zhou L.D., 2011. Analysis of eco-security carrying capacity on village scale in Miyun reservoir, Beijing[J]. Chinese Agricultural Science Bulletin, 23, 221-226(in Chinese).

[9] Lan Shengfang, Qinpei, Luhongfang et al. Emergy synthesis of ecological economic systems[M]. Beijing: Chemical industry Press, 2002 (in Chinese).

[10] Hu,Y.X., Zhou L.D., 2008. Evaluation of a demonstration fish farm using river water upreach of Miyun Reservoir, Beizhuang district, Beijing[J]. Acta Ecologica Sinica, 11,5675-5683(in Chinese).

[11] Mark T.B., Eliana B., 2002. Handbook of Emergy Evaluation, a compendium of data for emergy computation issued in a series of Folios\#3, Emergy of Ecosystems[M]. Gainesville: University of Florida, 32611-6450.

[12] Odum, H.T., 2000. Folio \#2: Emergy of Global Processes. Handbook of Emergy Evaluation. Center for Environmental Policy, University of florida, Gainesville, FL.

[13] Sherry L.B-W., 2002. Handbook of Emergy Evaluation, a compendium of data for emergy computation issued in a series of Folios\#4, Emergy of Florida agriculture[M]. Gainesville: University of Florida, 32611-6450.

[14] Yan M.C., 2001. Introduction to new ecological economics[M]. Beijing: China Zhigong Press. 